\title{
Single Treatment Approaches to Benign paroxysmal Positional Vertigo, Is It Enough?
}

\author{
Ahmed Abdel-Bassit* , Mohammed Elsaid ** and \\ Mohammed El-Beltagy*.
}

\author{
*Ear, Nose, and Throat Department, AlAzhar Faculty Of Medicine (Boys); and \\ **Ahmed Maher Educational Hospital.
}

\begin{abstract}
Bening paroxysmal positional vertigo (B.P.P.V) is one of the most common vestibular disorders.During the last years, it has been attributed to cupulolithiasis or canalithiasis of the posterior semicircular canal.This randomized study was done to determine the effectiveness of two different physical therapy approaches and to determine if a single treatment approaches is enough.In this prospective study 80 patients received either a single treatment based on the hypothesis that the vertigo and nystagmus of (B.P.P.V) are due to debris adhering to the cupula of posterior semicircular canal (cupulolithiasis), Semont maneuver, or a single treatment based on the hypothesis that the debris is free floating in the long arm of the posterior semicircular canal (canalithiasis), Epley maneuver.Treatment outcome was classified as asymptomatic, improved and no change.

Analysis of the results of this study revealed that at the end of the first session, out of 80 patients, 60(75\%) were asymptomatic ; 14(18\%) improved ;6(7\%) showed no change. Followup showed that 8 patients developed recurrence. After a second session to 22 patients (8 recurrences, 10 improved, 4 had no change) ,17 became asymptomatic.This means that at the end of the seconed session ,69 (86\%) of 80 patients became asymptomatic ; 9(11\%) of 80 patients improved ; $2(3 \%)$ of 80 patients showed no change.

In conclusion, this study shows that 1) The success rate of Semont's maneuver was up to $80 \%$, so it is much better than Epley maneuver $70 \%$ after the first session. 2)Recurrent cases are best treated with second session.
\end{abstract}

\section{Introduction}

Among vertiginous disorders, benign paroxysmal positional vertigo (B.P.P.V) is common. Schuknecht (1974) has proposed cupulolithiasis or canalithiasis as the principal mechanism. Calcium carbonate crystals from saccular or utricular otoconia impinge on the cupula of the posterior semicircular canal the most dependant portion of the vestibular labyrinth.

The first hypothesis, cupulolithiasis, proposes that degenerative debris (possibly fragments of otoconia from the utricle) adhere to the surface of the cupula of the posterior semicircular canal facing the utricle, making its gravity sensitive. This mechanism was first suggested by Schuknecht who found basophilic-staining deposits on the cupola of the posterior semicircular canal in two patients with a history of BPPV. The increased density of the cupula relative to the endolymph results in an inappropriate deflection of the cupula of the posterior canal when the head is moved while the posterior canal is in a plane parallel to the pull of gravity .Leading to vertigo, nystagmus, and nausea. Dislodging the debris from the cupula and expelling it into the vestibule should relieve the symptoms.

The second hypothesis, canalithiasis, (Hall et al.,1979 and Epley,1992) suggests that the degenerative debris does not adhere to the cupula of the posterior canal but rather is free floating in the endolymph of the long arm of the canal. When the head is moved into the provoking position, the debris sinks into the most dependent position in the canal, causing the 
endolymph to move away from the ampulla. This movement of the endolymph overcomes the inertia of the cupola, resulting in inappropriate exitation of the ampullary nerve of the canal. Furthermore, one can envision that moving the head in an appropriate sequence of orientations with respect to gravity could result in the debris moving out of the posterior semicircular canal, into the common crus, and subsequently into the vestibule. This would relieve the patient's symptoms.

The diagnosis is chiefly based on the basis of history and physical examination. Vertigo and nystagmus are elicited upon hallpike positioning, classically, there is a period of latency up to 5 seconds before nystagmus begins. Nystagmus is usually of the rotatory type,beating toward the underlying ear, although horizontal nystagmus may be present Vertigo increases in intensity and subsequently disappears within 40 seconds. Nystagmus is fatigable and should reverse direction when the upright position is assumed.

Positional vertigo of the benign paroxysmal type is distinguished by the usually brief duration of each attack and the absence of auditory symptoms.

It is should be differentiated from positional nystagmus due to central nervous system lesions, which has the main following criteria:

1. It occurs with no latent period.

2. The direction of nystagmus is variable.

3. The nystagmus persists while position is maintained.

4. The nystagmus persists with repeated testing.

There are several different exercise treatments have been developed as:

1. Brandt-Daroff exercise.

2. Semon et al and Toupet.

3. Epley.

In this study we use two different single treatment approaches. One treatment proposed by Semon et al and Toupet which based on the theory of cupulolithiasis and the other treatment, proposed by Epley, is based on canalithiasis.
The purpose of our study was to compare and determine the effectiveness of two different physical therapy approaches for benign paroxysmal positional vertigo.

\section{Subjects and methods}

80 patients were diagnosed as (B.P.P.V) after positive Dix-Hallpike maneuver.

There is a period of latency before the onset of vertigo and nystagmus. Nystagmus is usually of rotatory type, beating toward the underlying ear.

Vertigo and nystagmus lasting less than 60 seconds.

-Patients with disorders of the centeral nervous system were excluded from the study.-Patients age ranged from 32 to 68 years. There were 50 women -30 men.

-Patients were randomly assigned to receive the Semont maneuver $(n=40)$ or the modified Epley maneuver $(\mathrm{n}=40)$.

In the Semont maneuver Semont et al., (1988) the patient sat on the side of a treatment table. The head turned 45 degrees away from the affected ear and then the patient was rapidly moved into a sidelying position. After 4 minutes, the patient was rapidly moved, en bloc, through the initial sitting position to the opposite sidelying position. As the patient was moved, the original alignment of the head on body was maintained by the therapist. The patient was kept in the second sidelying position for 4 minutes.If during the first minute in this position, the patient did not experience vertigo, the patients head was jostled briskly through a short range of motion two or three times in an attempt to dislodge the debris, At the end of 4 minutes, the patient was slowly moved from sidelying to a sitting position. The patients were told to keep their head in an upright (vertical) position for 48 hours. Specifically, they were told not to bend over, look up or down with their head. For the subsequent 5 days, patients were allowed to lie down but not on the affected side.In the modified Epley maneuver, Epley (1992), the patient's head was turned 45 degrees toward the affected side. The patient was then moved from a 
sitting position into the Hallpike-Dix position. After 4 minutes, the head was extended further and then slowly turned 100 degrees, over the course of 1 minute, to the opposite Hallpike-Dix position. After4 minutes, the patient slowly sat up. At that time, the patient was given the same instructions as the patients treated with the Semont maneuver.

One to two weeks after the treatment, patients were either reevaluated in the clinic or reported the outcome of the treatment by telephone. Treatment outcome was classified as (1) asymptomatic, no vertigo and, if seen in the clinic, no nystagmus. (2) improved, the patients subjectively rated their condition as $70 \%$ improved or better and had returned to previously avoided activities; nystagmus was still present. (3) no change, no change in symptoms or the patient stated that symptoms had improved less than $70 \%$, nystagmus still present. Forty-eight percent of the patients have had follow-up visits by telephone between 1 to 18 months after the initial treatment.

\section{Results}

- All patients in this study were followed-up over 18 months after the initial treatment.

- There was no significant difference in the age or in the distribution of men and women in the two treatment groups.

- The average age of the patients was 50+18 years. There were 50 women and 30 men in the study.

- The group treated with Semont's maneuver $80 \%(32 / 40)$ of the subjects have no vertigo after the first treatment, $15 \%$ $(6 / 40)$ were improved, and 5\% (2/40) had no change in the symptoms.

- The group treated with Epley maneuver $70 \%(28 / 40)$ had no vertigo, $20 \%(8 / 40)$ were improved, and $10 \%(4 / 40)$ had no change.

- Eight out of the 60 patients who had no vertigo after treatment had recurrent osition induced vertigo confirmed by the HallpikeDix test, complained of a slight and very brief off-balance sensation when moved into the Hallpike-Dix position but had no nystagmus. They were asymptomatic after the second session.
- Ten out of the 14 patients who were improved after the first treatment were treated a second time. Seven of them became asymptomatic.

- Six patients without change after a single treatment, four received further treatment, and two were unavailable for follow-up.

The four retreated patients, two were asymptomatic, and two were improved after further treatment.

22 patients received second session:

17 out of them had no vertigo, 2 improved.

Semont's maneuver:

\begin{tabular}{|l|l|l|}
\hline Total No. & 40 & \\
\hline Average age & $52+-18$ & \\
\hline Male & 15 & \\
\hline Female & 25 & \\
\hline Cured & 32 & $80 \%$ \\
\hline Improved & 6 & $15 \%$ \\
\hline No change & 2 & $5 \%$ \\
\hline
\end{tabular}

Epley Maneuver:

\begin{tabular}{|l|l|l|}
\hline Total No. & 40 & \\
\hline Average age & $52+-18$ & \\
\hline Male & 15 & \\
\hline Female & 25 & \\
\hline Cured & 28 & $70 \%$ \\
\hline Improved & 8 & $20 \%$ \\
\hline No change & 4 & $10 \%$ \\
\hline
\end{tabular}

Results after second session:

\begin{tabular}{|l|l|l|}
\hline Cured & 69 & $86.25 \%$ \\
\hline Improved & 7 & $11.25 \%$ \\
\hline No change & 4 & $2.5 \%$ \\
\hline
\end{tabular}

\section{Discussion}

Analysis of the results of this study revealed that at the end of the first session:

- $60(75 \%)$ out of 80 patients were asymptomatic

$-14(18 \%)$ out of 80 patients were improved

- $6(7 \%)$ outof 80 patients had no change.

Follow -up showed that the number of recurrence was 8 patients. 
After a second session to 22 patients (8 recurrences, 10 improved, 4 had no change).

17 developed asymptomatic.

As noted from above that at the end of the seconed session:

$-69(86 \%)$ of 80 patients were asymptomatic.

$-9(11 \%)$ of 80 patients were improved.

$-2(3 \%)$ of 80 patients had no change.

Theoretically, the Semont maneuver would be effective primarily in patients with adherent debris to the cupula of the posterior semicircular canal also it may be effective when the debris is free floating in the long arm of the posterior canal. For patients with debris free floating in the long arm of the posterior semicircular canal, maintaining the initial position on the affected side would allow the debris to move down into the most dependent location in the canal. When the patient's position is changed rapidly to lying on the opposite side, the debris could fall into the common crus, therefore relieving the patient's symptoms occurs. There are several reasons why the Semont maneuver was not effective in all patients treated. First, it is possible that some but not all of the debris was jarred free from the cupula during the treatment. Second, in some patients in whom the debris was in the long arm of the posterior canal, the Semont maneuver may not have successfully displaced the debris into the common crus.

The modified Epley maneuver presumably is effective only for those patients in whom the debris is free floating in the long arm of the posterior semicircular canal and would not be effective in patients in whom the debris is adhering to the cupula. One reason the modified Epley maneuver may not have been effective in all patients treated may be because of the pathogenesis of the disorder. That is, for some patients, the pathogenesis of BPPV is cupulolithiasis and for others, the basis is canalithiasis. Patients who improved but were not "cured" after a single treatment may have debris adhering to the cupula attached located in the long arm of the posterior canal.
Another explanation for why neither the Semont nor the modified Epley treatment was successful in all patients may be that the maneuvers removed some, but not all, of the debris. There is considerable variation in the orientation of the posterior semi-circular canal among individuals. It is possible that, in some patients, debris that was free floating in the long arm of the posterior canal would not be moved sufficiently toward the common crus during the maneuver and, therefore, would move back into the posterior canal when the patient sat up.

Our results on the efficacy of single treatment using the Semont maneuver $(80 \%$ asymptomatic) similar to those reported by Semont for one treatment $(85 \%)$ but are differ from those reported by Semont et al.,(1988) and by Pagnini et al .,(1989).

$(68 \%)$. The difference between our results and those of Semont et al and by Pagnini may reflect some variation in the way that maneuvers were performed or in the definition of cure. A. Semont, PhD, suggests that the treatment should be repeated within the same session to assure the displacement of the debris. We performed only one maneuver during any given treatment session. Another possibility may be the time from onset of the symptoms to time of treatment. Semont et al. (1988).do not state the time from onset of symptoms to treatment of the patient, and their group may have included a larger proportion of patients who were seen during the earlier period in whom spontaneous recovery was more. likely' to occur

Our results for the modified Epley maneuver (70\% asymptomatic) also differ from those reported for the treatment by Epley" (80\% asympto-matic). There are several differences between our treatment and Epley's. Like Semont et al, Epley states that he may repeat the maneuver several times during a single treatment session while we studied the effect of a single maneuver. Harvey et al. (1994) re-ported on 25 patients: $44 \%$ had a complete early response, $24 \%$ improved and $32 \%$ had failures. In a randomized prospective study Lynn et al. (1995) demonstrated an 89\% 
response rate after 1 treatment as compared with a $26.7 \%$ response rate in a control group.Recently, Asaw-arichiginda et al. (2000), Balatsouras et al. (2000) and Nunez et al. (2000) have recommended the use of repeat treatment management of BPPV.

However, the number of repeated sessions required to cure a patient with BPPV was variable among various studies. Nunez et al. (2000) stated that two treatment sessions are usually adequate to achieve the best outcome. In our study, patients who did not respond to the second treatment session (no change) were considered to have failure results.

\section{Conclusion}

The success rate of Semont's maneuver was up to $80 \%$, so it is much better than Epley maneuver $70 \%$ after the first session.

Our study recommends that recurrent cases are best treated with second session.

\section{References}

1. Asawarichingda, S., Isipradit, P., Snidrongs, K. and Supiyaphun, P. (2000): Efficacy of canalith repositioning for BPPV. Ear, Nose \&Throat Journal, 69:732 737.

2. Balatsouras, D., Kloutsos,G., Georgopoulas,

V.,Protopupas,D.,Aspris, A., Loretzas, D., Nikelakapoulas, A., and Edmonou., C.(2000): Benign paroxysmal positional vertigo. Treatment with mod-ified Epley maneuver. Hellenic Otorhino laryngology Head and Neck Surgery, 21: 32-37.
3. Epley, J. M. (1992): The canalith repositioning procedure for treatment of benign paroxysmal positional vertigo. Otolaryngology-Head and Neck surgery, 107:399 - 404.

4. Hall,S.F.,Ruby,R.R.F.and McClure,J.A (1979): The mechanisms of benign paroxysmal vertigo. Journal of Otolarygology, 8:151 - 158.

5. Harvey, S.A., Ham, T. C.and Adamiec, L.C.(1994): Modified liberatory maneuver :effective treatment for benign paroxy-smal positional vertigo. Laryngoscope, 104:1206 -1212.

6. Lynn, S., Poole, A. and Rose, D. (1995): Randomized trial of the canalith repositioning procedurs. Otolaryngology- Head and Neck Surgery, 113: 714-719.

7. Nunez, R. A. Cass, S. P., and Furman J. M (2000) Short-and long-term outcomes of canalith repositioning for benign paroxysmal positional vertigo Otolaryng-ology.Head and Neck Surgery, 122:647 -652.

8. Pagnini P,vannucchi P,Vicini.(1989): $\quad$ Particle repositioing maneuver for benign paroxysmal positional vertigo. Annals of Otology, Rhinology and laryngology, 102:325-331

9. Semont,A. Freyss, G.and Vitte,E. (1988) Curing the (B.P.P.V) with a liberatory maneuver.Advanced Otorhinolaryngology, 42: 290 293.

10. Shuknecht , H.F.(1969): Cupulolithiasis. Archives of Otolaryngology, 90: 765 - 769 . 


\title{
جلسة واحدة لعلاج نوبات مرض الدوار الوضعي الحميد هل تكفي؟
}

\author{
* أحمد عبد الباسط ـ * * محمد السيا * * محمد البلتاجى

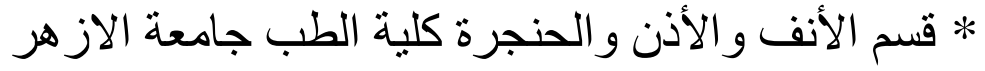

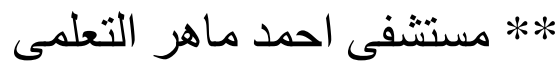

يعتبر مـرض الدوار الوضـعي الحميد النـاتج عن تغييـر وضـع المـريض هـو أكثر

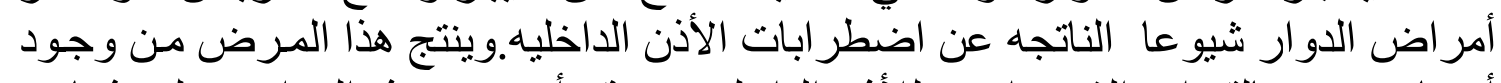

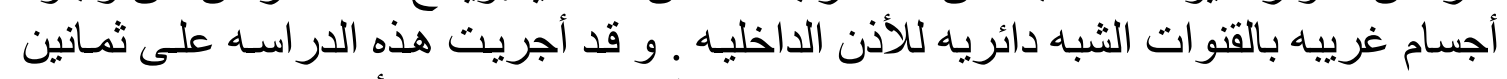

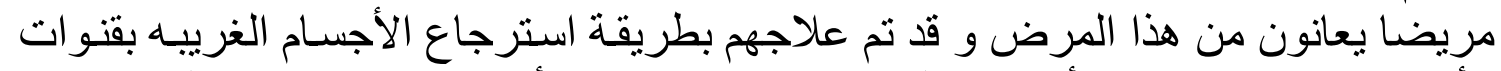

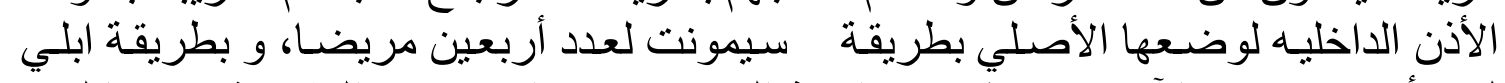

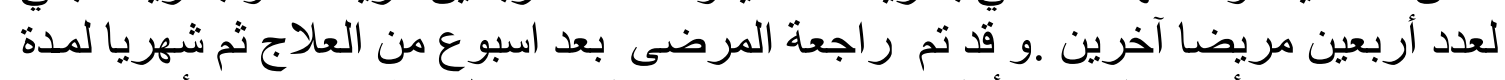

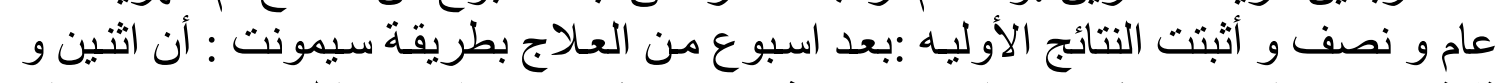

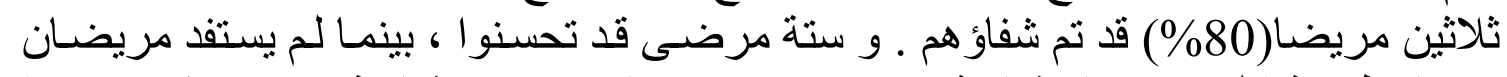

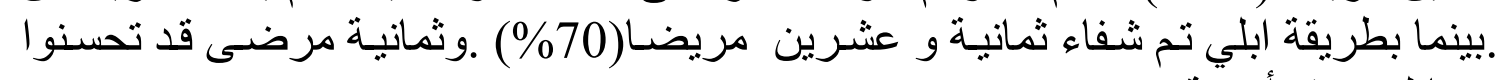

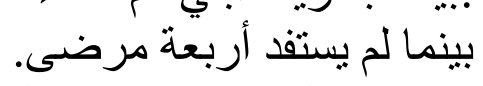

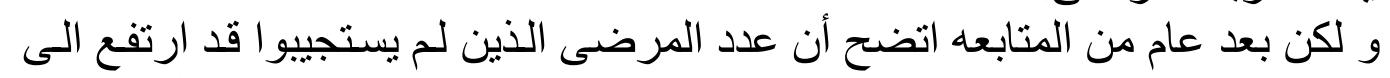

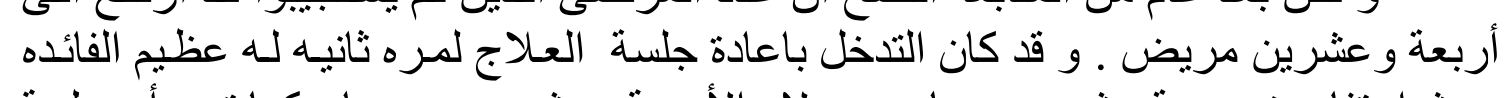

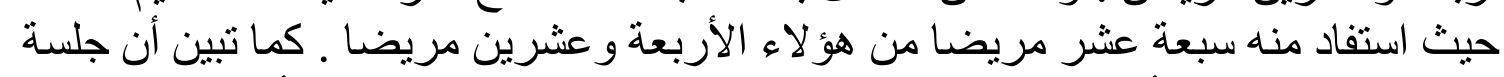

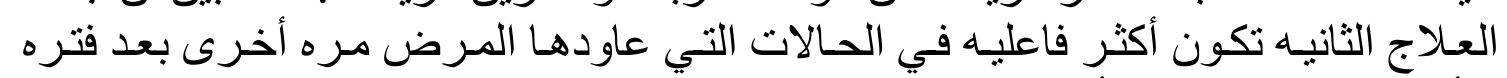

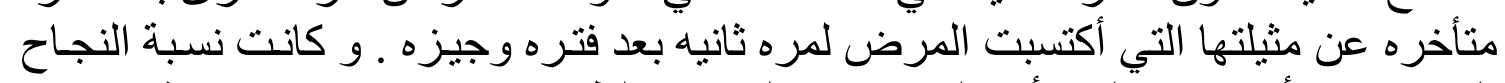

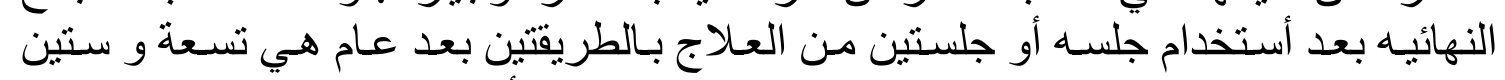

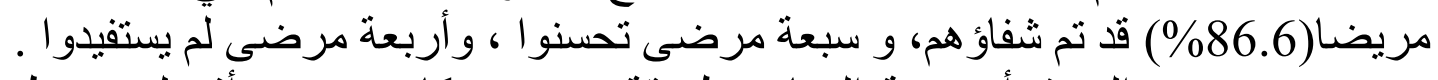

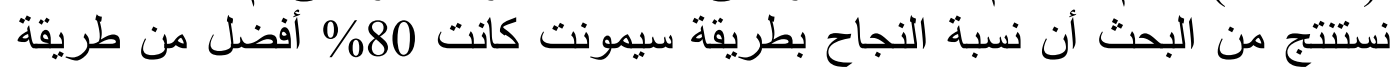

ابلي570\% وكذللك نوصي أن جلسة ثانية للغير حاصلين على نتيجة ايجابية من جلسة واحدة تعطي نتيجة أكثر فاعلية. 
Single Treatment Approaches to Benign........... 